\title{
Calculation of the Maximum Number of Cutting Finite Fields in the Multi-dimensions
}

\author{
Erick C. Huang ${ }^{1}$, Sharon S. Huang ${ }^{1} \&$ Cheng-Hua Tsai ${ }^{2}$ \\ ${ }^{1}$ Morrison Academy, Taichung, Taiwan \\ ${ }^{2}$ Department of Mathematics, Taichung Municipal Taichung First Senior High School, Taichung, Taiwan \\ Correspondence: Erick C. Huang, Morrison Academy, Taichung, Taiwan 43715.
}

Tel: 1-886-426-762-528. E-mail: cs2777726@gmail.com

Received: May 11, 2017 Accepted: June 26, 2017 Online Published: July 3, 2017

doi:10.5539/jmr.v9n4p49 URL: https://doi.org/10.5539/jmr.v9n4p49

\begin{abstract}
The original problem that serves as a basis for this project comes from an American contest (PUMaC, 2014) regarding the maximum amount of enclosed spaces given a limited number of cuts on an infinite plane. In this study, we explore the same problem and extend it in the context of $m$ dimensions given $n(m-1)$ dimensional cuts using the recursive relationship of finite cuts and enclosed spaces in lower dimensions. Once the general formula of $f(m, n)$ was proven for $m$ dimensions, an Euler's inspired formula was used to check the accuracy of the formula in two and three dimensions. The Euler's formula also allowed us to derive the formula for the maximum number of unenclosed spaces in three-dimensional $F(3, n)$. The results are as follows:
\end{abstract}

(a) In an infinite $m$ dimensional space $(m \in N, m \geq 2)$, given $n(m-1)$-dimensional cuts (all of which are infinite fields), the maximum number of enclosed fields $f(m, n)$ is $f(m, n)=C_{m}^{n-1}$ where $m, n \in N$ and $m \leq n$

(b) In a $m$ dimensional ( $m \in N, m \geq 2$ ) space, given $n(m-1)$ dimensional cuts, there will be a maximum of $F(m, n)$ enclosed or infinite field total, where $F(m, n)$ can be expressed as:

(1) If $m$ is odd, $F(m, n)=C_{m}^{n+1}+C_{m-2}^{n+1}+C_{m-4}^{n+1}+\cdots+C_{1}^{n+1}$, and $m, n \in N, \quad m \leq n$

(2) If $m$ is even, $F(m, n)=C_{m}^{n+1}+C_{m-2}^{n+1}+C_{m-4}^{n+1}+\cdots+C_{0}^{n+1}$, and $m, n \in N, m \leq n$

Keywords: finite field, recursive relations, Euler characteristic

\section{Introduction}

\subsection{Research Motivation}

The mathematical project course is to explore an interesting mathematics competition problem from PUMaC (PUMaC, 2014), on the subject that is described as follows:

Assume you have a magical pizza in the shape of an infinite plane. You have a magical pizza cutter that can cut in the shape of an infinite line, but it can only be used 14 times. To share with as many of your friends as possible, you cut the pizza in a way that maximizes the number of infinite pieces (the infinite pieces have infinite mass, so you can't lift them up). How many finite pieces of pizza do you have?

The interesting study of the number of cuts it takes to make a given amount of closed areas has long been studied (Simone, C.D., Rinaldi, G.., 2008). Problems pertaining to the maximum number of areas that can be made with a given number of cuts have shown up in previous mathematics contests (Puzzling Stack Exchange, 2014). This simple math problem gives rise to an interesting question. Since all previously found problems were set in a two dimensional condition, we wondered if the number of finite cuts needed to define a given number of closed areas change in different dimension conditions. This study investigates the relationship between the numbers of finite cuts required in an infinite plane set in the condition of one, two, three, and even $n$ dimensions.

Through the method of finding the recursive relationship of cuts and closed area within each dimension and the recursive relationship between one dimension to the next, a formula for $n$ dimensions given a certain number of areas was determined to calculate the number of finite cuts needed. 


\subsection{Research Purposes}

(a) Find the general form of $f(2, n)$ and $f(1, n)$.

(b) Using the same methods to derive the general forms of $f(2, n)$ and $f(1, n)$., find a mathematical model for the general form of $f(m, n)$.

(c) Using the established mathematical model for the general form of $f(m, n)$, find the general form of $F(m, n)$.

(d) Establish another mathematical model using Euler's Polyhedron Formula (Lazy caterer's sequence, 2017) to confirm the general forms of $f(2, n), f(3, n), F(2, n)$, and $F(3, n)$. In addition, we investigate other extensions of the original problem.

\section{Research Methods and Processes}

\subsection{Key Word Definitions}

Enclosed and infinite field in $m$ dimensions: Any area that is bounded by boundaries (whether they are lines, planes, etc.) in $m$ dimensions is defined as enclosed. All other areas are defined as infinite. For example, a segment in one dimension is regarded as an enclosed field, and a ray in one dimension is regarded as infinite. Similarly, a triangle in two dimensions is regarded as enclosed and a half plane (infinite plane divided by a line) is regarded as an infinite object.

$f(m, n)$ : denotes the maximum number of enclosed fields that result from $n$ cuts (which are $m-1$ dimensional) in a $m$ dimensional space

$F(m, n)$ : denotes the maximum number of fields, including enclosed fields and infinite fields that result from $n$ cuts (which are $m-1$ dimensional) in a $m$ dimensional space

Connected Enclosed Graphs:

(1) A connected enclosed graph in one dimension consists of an infinite line divided by $n$ points (cuts) into $n-1$ finite segments (enclosed areas) and two rays, each on one end.

(2) A connected enclosed graph in two dimensions consists of an infinite plane divided by $\mathrm{n}$ intersecting infinite lines (cuts), creating both enclosed areas and infinite areas.

(3) A connected enclosed graph in three dimensions consists of an infinite three dimensional space divided by infinite planes (cuts), creating both enclosed objects and infinite objects.

(4) A connected enclosed graph in $\mathrm{m}$ dimensions consists of an infinite $\mathrm{m}$ dimensional space divided by infinite $\mathrm{m}-1$ dimensional fields (cuts), creating both enclosed fields and infinite fields.

Modified Euler's Equation:

(1) Modified Euler's Equation in one dimension: The equation V-E =1, modified from Euler's Polyhedral Formula, is satisfied in a connected enclosed graph in one dimension, where $\mathrm{V}$ is the number of vertices and $\mathrm{E}$ is the number of edges in the connected enclosed graph.

(2) Modified Euler's Equation in two dimensions: The equation V-E+F =1, modified from Euler's Polyhedral Formula, is satisfied in a connected enclosed graph in two dimensions, where $\mathrm{V}$ is the number of vertices, $\mathrm{E}$ is the number of edges, and $\mathrm{F}$ is the number of faces in the connected enclosed graph.

(3) Modified Euler's Equation in three dimensions: The equation V-E+F-S $=1$, modified from Euler's Polyhedral Formula, is satisfied in a connected enclosed graph in three dimensions, where $\mathrm{V}$ is the number of vertices, $\mathrm{E}$ is the number of edges, $\mathrm{F}$ is the number of faces, and $\mathrm{S}$ is the number of finite solids in the connected enclosed graph.

\subsection{Solution of the Original Problem and Generalization of $f(2, n)$}

\subsubsection{An Investigation of the Original Problem.}

Problem:

There is a magical pizza in the shape of an infinite plane. If you can cut this magical pizza with a magical pizza cutter in the shape of an infinite line, and it can only be used 14 times. How many finite pieces of pizza do you have at most?

\section{[Solution]}

Consider a circle $\mathrm{C}$ that contains all points of intersection created by the cuts. It is clear that all infinite pieces of pizza cannot be contained in $\mathrm{C}$ and there are no finite pieces outside $\mathrm{C}$. Hence, the number of infinite pieces is equal to the sectors outside of C. Since each cutting line passes through C, there will be $2 \times 14=28$ infinite sectors outside C.

We note that the last line can intersect with at most all of the 13 of the preexisting lines, which will divide that last line 
into 15 distinct parts. Since each part divides one existing area into two, there can be at most 15 new areas. In addition, the preexisting infinite plane must be taken into account. Hence, with 14 lines there will be a total of $\frac{14 \times 15}{2}+1=106$ areas at most and $106-28=78$ of them are finite.

The maximum number of areas can be obtained by drawing lines such that no two lines are parallel and no three lines are concurrent. In other words, each line must intersect all others at distinct points and thus each line will have $n+1$ distinct segments that make $n+1$ new areas. Hence this construction will have 106 areas, 78 of them is finite.

2.2.2 Extension of the Problem to a Generalized Form of $f(2, n)$

Imagine an infinite pizza that is cut with $n$ infinite lines. The maximum number of enclosed pizza areas resulting from

the $n$ cuts is denoted as $f(2, n)$ pieces of pizza, where $f(2, n)=\frac{(n-1)(n-2)}{2}$.

\section{[Proof]}

Notice that the two extreme ends of this $n$th line are border to unenclosed spaces, the additional number of enclosed spaces that are produced by the $n$th cut is $n-2$. In other words, we conclude that $f(2, n)$ will have $n-2$ more enclosed spaces than $f(2, n-1)$. Thus, we express the observations by the following:

$$
\begin{aligned}
& \left\{\begin{array}{l}
f(2,1)=0, f(2,2)=0, f(2,3)=1 \\
f(2, n)=f(2, n-1)+(n-2)
\end{array}\right. \\
& f(2, n)-f(2, n-1)=n-2 \\
& f(2,3)-f(2,2)=1 \\
& f(2,4)-f(2,3)=2 \\
& f(2, n)-f(2, n-1)=n-2
\end{aligned}
$$

Summing all the equations, we get

$$
\begin{aligned}
& f(2, n)-f(2,2)=1+2+3+\cdots+(n-2)=\frac{(n-1)(n-2)}{2} \\
& \Rightarrow f(2, n)=\frac{(n-1)(n-2)}{2} .
\end{aligned}
$$

Using the formula from $f(2, n)$, we strive to calculate the general formula for $f(1, n)$.

Now let us go back and look at in one dimensional infinitely long object.

\section{Case 1: 1 cut}

If we cut once, we would not get a smaller piece.

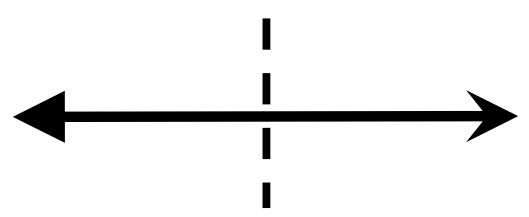

We can conclude that $f(1,1)=0$

Case 2: 2 cuts

It is immediately obvious that we have cut out a piece( shown in red in the figure below) from the 1 dimensional object 
with 2 cuts.

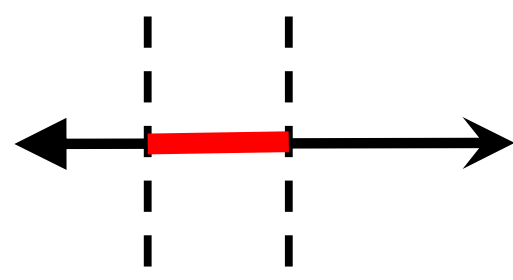

We can then say that $f(1,2)=1$

Case 3: 3 cuts

As observed from the figure below, making 3 cuts in a 1 dimensional object results in 2 pieces of that object( as shown in red).

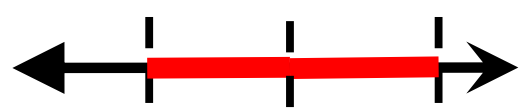

We can then get that $f(1,3)=2$

\section{Case n: $n$ cuts}

Let's look at how many pieces are made after cutting the 1-D object with $\mathrm{n}$ cuts.

We observe that every 2 cuts make a piece so we conclude that $f(1, n)=n-2+1=n-1$

From the formulas of $f(1, n)$ and $f(2, n)$ previously proved, we would like to derive the general formula for $f(m, n)$. However, a stricter mathematical model needs to be constructed to find the formula for $f(m, n)$ instead of relying on a recursive pattern.

The next section focuses on finding and constructing a mathematical model.

\subsection{Finding a Mathematical Model and a General Formula for $f(m, n)$}

Previously, the formula was found through the use of recursive relationships. Now, the recursive relationships are explained in hopes of finding a mathematical model that would allow us to calculate $f(m, n)$.

\subsubsection{Establishing the Mathematical Model}

Since $f(1, n)$ represents the maximum number of enclosed fields in one dimension given $\mathrm{n}$ cuts, $f(1, n)$ represents the number of segments created by $\mathrm{n}$ points on the infinite line. $\mathrm{F}(1, \mathrm{n})$ will fulfill the following:

$$
\left\{\begin{array}{l}
f(1,1)=0 \\
f(1, n)-f(1, n-1)=1, n \geq 2, n \in N
\end{array}\right.
$$

\section{[Explanation]}

When $n=1$, there will only be one point on the infinite one dimensional line which divides the line into two rays, neither of which are considered enclosed. Therefore, $f(1,1)=0$. Similarly, when $n=2$, there will be two points on the infinite one dimensional line, dividing the line into two rays and a segment. Thus, $f(1,2)=1$.

As we have established the first two terms of $f(1, n)$, we would like to explore the recursive relationships in the series between $f(1, n)$ and $f(1, n-1)$. We first observe that an additional point will result in an additional segment, so $f(1, n)-f(1, n-1)=1$

Therefore, we can conclude that

$$
\left\{\begin{array}{l}
f(1,1)=0 \\
f(1, n)-f(1, n-1)=1, n \geq 2, n \in N
\end{array}\right.
$$

\subsubsection{Moving on to Two Dimensions}


Since $f(2, n)$ represents the maximum number of enclosed fields in two dimensions given n cuts, $f(2, n)$ represents the number of segments created by $\mathrm{n}$ points on the infinite line. $f(2, n)$ will fulfill the following:

$\left\{\begin{array}{l}f(2,1)=0 \\ f(2, n)-f(2, n-1)=f(1, n-1), n \geq 2, n \in N\end{array}\right.$

\section{[Explanation]}

When $n=1$, there will only be one line dividing an infinite plane into two infinite fields. Thus, $f(2,1)=0$. Similarly, when $n=2$, the infinite plane will be divided by two lines, forming four infinite fields. Thus, $f(2,2)=0$.

After establishing the first two terms of $f(2, n)$, we explore the recursive relationships in the series between $f(2, n)$ and $f(2, n-1)$. To obtain the maximum number of enclosed areas given $\mathrm{n}$ cuts, every cut must intersect with every other cut, which means the nth cut will have n-1 intersections. This will create an additional $f(1, n-1)$ segments, each of which is bordering a newly created enclosed field. Therefore, $f(2, n)$ will have $f(1, n-1)$ more enclosed fields than $f(2, n-1)$. All the observations are summarized below:

$$
\left\{\begin{array}{l}
f(2,1)=0 \\
f(2, n)-f(2, n-1)=f(1, n-1), n \geq 2, n \in N
\end{array}\right.
$$

From the explanations above, the recursive rule is a very helpful tool and a great mathematical model for obtaining a general formula. Thus, we continue to use similar methods to construct a model for the general formula.

\subsubsection{Mathematical Model 1}

In $\mathrm{m}$ dimensions, the expression $f(\mathrm{~m}, n)$ represents the maximum number of enclosed fields in $\mathrm{m}$ dimensions given $\mathrm{n}$

cuts. $f(\mathrm{~m}, n)$ will fulfill the following: $\left\{\begin{array}{l}f(m, 1)=0 \\ f(m, n)-f(m, n-1)=f(m-1, n-1), n \geq 2, n \in N\end{array}\right.$

\section{[Explanation]}

When $n=1$, there will only be one m-1 dimensional cut dividing an infinite field into two infinite fields. Thus, $f(m, 1)=0$. Similarly, when $n=2$, the infinite field will be divided by two cuts of $\mathrm{m}-1$ dimensions, forming infinite fields but no enclosed spaces. Thus, $f(m, 2)=0$. As we have established the first two terms of $f(2, n)$, we explore the recursive relationships in the series between $f(2, n)$ and $f(2, n-1)$.

It is observed that an additional $\mathrm{m}-1$ dimensional cut in $\mathrm{m}$ dimensional space would cause an addition of $f(m-1, n-1) m-1$ dimensional cuts, which borders the newly formed $f(m-1, n-1)$ spaces. Thus, $f(m, n)-f(m, n-1)=f(m-1, n-1)$.

We summarize the findings: $\left\{\begin{array}{l}f(m, 1)=0 \\ f(m, n)-f(m, n-1)=f(m-1, n-1), n \geq 2, n \in N\end{array}\right.$

Using the same methods and generalized form from above, we solve for generalized formulas for $f(3, n)$ and $f(4, n)$.

(1) Derivation of $f(3, n)$ formula

\section{[Investigation]}

Moving on to three dimensions, we denote the number of enclosed areas as $f(3, n)$ 'where $n$ is the number of cuts. Through experimentation, we know that the minimum number of cuts needed to create an enclosed area is four cuts resulting in a tetrahedral shape. So 


$$
\begin{aligned}
& f(3,0)=0 \\
& f(3,1)=0 \\
& f(3,2)=0 \\
& f(3,3)=0 \\
& f(3,4)=1
\end{aligned}
$$

A question arises. How many additional enclosed areas will an additional cut create? To maximize the number of areas each successive cut creates, the successive cut must intersect all the existing cuts. That means that the nth cut will have n-1 intersections. However, in three dimensions, cuts are planes. The nth plane will intersect with n-1 other planes, and these intersections are lines! In other words, the nth plane will create n-1 lines, which creates n-1 enclosed areas. We symbolize our findings by the following recursive rule:

$f(3, n)=f(3, n-1)+f(2, n-1) \rightarrow f(3, n)-f(3, n-1)=f(2, n-1)$

We summarize by expressing the following:

$$
\begin{aligned}
& \left\{\begin{array}{l}
f(3,1)=0, f(3,2)=0, f(3,3)=0 \\
f(3, n)-f(3, n-1)=f(2, n-1)
\end{array}\right. \\
& f(3, n)-f(3, n-1)=\frac{(n-2)(n-3)}{2} \\
& f(3,2)-f(3,1)=0 \\
& f(3,3)-f(3,2)=0 \\
& f(3,4)-f(3,3)=0 \\
& f(3,5)-f(3,4)=1 \\
& f(3, n)-f(3, n-1)=\frac{(n-2)(n-3)}{2}
\end{aligned}
$$

To get a formula for $\mathrm{f}(3, \mathrm{n})$, we add up all previous $\mathrm{n}$ terms to get

$$
\begin{aligned}
& f(3, n)-f(3,1)=0+0+1+\cdots+\frac{(n-2)(n-3)}{2}=\sum_{k=1}^{n-1} \frac{(k-1)(k-2)}{2} \\
& \Rightarrow f(3, n)=\frac{(n-1)(n-2)(n-3)}{6} . \\
& \text { (2) Derivation of } f(4, n) \text { formula }
\end{aligned}
$$

\section{[Investigation]}

Since $f(4, n)$ represents the maximum number of enclosed fields in four dimensions given $\mathrm{n}$ three-dimensional cuts, it is obvious that $f(4,1)=0, f(4,2)=0$, and that

$$
\left\{\begin{array}{l}
f(4,1)=0, f(4,2)=0, f(4,3)=0 \\
f(4, n)-f(4, n-1)=f(3, n-1)
\end{array}\right.
$$


From the generalized formula of $f(3, n)$, we substitute $f(3, n-1)$ in the recursive equation to get

$f(4, n)-f(4, n-1)=\frac{(n-2)(n-3)(n-4)}{6}$

Next, we list out the recursive equations.

$$
\begin{aligned}
& f(4,2)-f(4,1)=0 \\
& f(4,3)-f(4,2)=0 \\
& f(4,4)-f(4,3)=0 \\
& f(4,5)-f(4,4)=1 \\
& f(4, n)-f(4, n-1)=\frac{(n-2)(n-3)(n-4)}{6}
\end{aligned}
$$

Summing, we get

$$
\begin{aligned}
& f(4, n)-f(4,1)=0+0+1+\cdots+\frac{(n-2)(n-3)(n-4)}{6}=\sum_{k=1}^{n-1} \frac{(k-1)(k-2)(k-3)}{6} \\
& \Rightarrow f(4, n)=\frac{(n-1)(n-2)(n-3)(n-4)}{24} .
\end{aligned}
$$

\subsection{4 $f(m, n)$ Prediction Formula}

From previous proofs of general formulas in one, two, three and four dimensions, we re-express these formulas below to show a more obvious pattern, using the form of $C_{m}^{n}$

$$
\begin{aligned}
& f(1, n)=n-1=C_{1}^{n-1}, n \in N \\
& f(2, n)=\frac{(n-1)(n-2)}{2}=C_{2}^{n-1}, n \in N \\
& f(3, n)=\frac{(n-1)(n-2)(n-3)}{6}=C_{3}^{n-1}, n \in N \\
& f(4, n)=\frac{(n-1)(n-2)(n-3)(n-4)}{24}=C_{4}^{n-1}, n \in N
\end{aligned}
$$

There's an evident pattern for the generalized form. Applying that pattern, the general formula is predicted below

$$
f(m, n)=\frac{(n-1)(n-2)(n-3) \cdots(n-m)}{m !}=C_{m}^{n-1}, m, n \in N, m \leq n .
$$

\section{[Proof]}

Proof by induction: 
(1) When $m=1$, the equation $f(1, n)=n-1=C_{1}^{n-1}$ holds.

(2) Let us assume that when $m=k$ the equation $f(k, n)=C_{k}^{n-1}$ holds. We would like to prove that when $m=k+1$, the equation $f(k+1, n)=C_{k+1}^{n-1}$ holds.

When $m=k+1$, we have

$$
\begin{aligned}
& f(k+1, n)=[f(k+1, n)-f(k+1, n-1)]+[f(k+1, n-1)-f(k+1, n-2)]+\cdots+ \\
& {[f(k+1,2)-f(k+1,1)]+f(k+1,1)} \\
& =f(k, n-1)+f(k, n-2)+f(k, n-3)+\cdots+f(k, 1)+f(k+1,1) \\
& =C_{k}^{n-2}+C_{k}^{n-3}+C_{k}^{n-4}+\cdots+C_{k}^{0}+0 \\
& =\sum_{i=1}^{n-2} C_{k}^{i}==\sum_{i=1}^{n-2} \frac{(i)(i-1)(i-2) \cdots(i-k+1)}{k !} \\
& =\frac{(n-1)(n-2)(n-3) \cdots(n-k-1)}{(k+1) !}=C_{k+1}^{n-1} .
\end{aligned}
$$

Our predicted formula has been proved and can be established as a theorem.

\section{[Theorem 1]}

In an infinite $m$ dimensional space ( $m \in N, m \geq 2$ ), given $n(m-1)$-dimensional cuts (all of which are infinite fields), the maximum number of enclosed fields $f(m, n)$ is $f(m, n)=C_{m}^{n-1}$ where $m, n \in N$ and $m \leq n$.

\subsection{Finding the General Formula for $F(m, n)$}

Using the recursive method that was used to obtain $f(m, n)$, the general formula for $F(m, n)$ was found. $F(m, n)$ represents the maximum number of areas created, infinite or finite, given $\mathrm{n}$ cuts in $\mathrm{m}$ dimensions.

\section{[ $F(m, n)$ Recursive Mathematical Model 1]}

In $\mathrm{m}$ dimensions, the expression $F(\mathrm{~m}, n)$ where $m \in N, m \geq 2$, represents the maximum number of enclosed fields in $\mathrm{m}$ dimensions given $\mathrm{n}$ cuts. $F(\mathrm{~m}, n)$ will fulfill the following:

$$
\left\{\begin{array}{l}
F(m, 1)=2 \\
F(m, n)-\mathrm{F}(m, n-1)=F(m-1, n-1), n \geq 2, n \in N
\end{array} .\right.
$$

\section{[Explanation]}

When $n=1$, there will only be one $\mathrm{m}-1$ dimensional cut dividing an infinite field into two infinite fields. Thus, $F(m, 1)=2$. Similarly, when $n=2$, the infinite field will be divided by two cuts of $m-1$ dimensions, forming infinite fields. Thus, $F(m, 2)=4$. After establishing the first two terms of $F(2, n)$, we explore the recursive relationships in the series between $F(2, n)$ and $F(2, n-1)$.

It is observed that an additional $\mathrm{m}-1$ dimensional cut in $\mathrm{m}$ dimensional space would cause an addition of $F(m-1, n-1) m-1$ dimensional cuts, which border the newly formed $F(m-1, n-1)$ spaces. Thus, $F(m, n)-F(m, n-1)=F(m-1, n-1)$. 
Therefore, we summarize the findings: $\left\{\begin{array}{l}F(m, 1)=2 \\ F(m, n)-\mathrm{F}(m, n-1)=F(m-1, n-1), n \geq 2, n \in N\end{array}\right.$

Using the same methods and generalized form from above, we solve for generalized formulas for $F(1, n), F(2, n)$, $F(3, n)$ and $F(4, n)$.

\subsection{1 $F(1, n)$ General Form}

\section{[Investigation]}

Using the mathematical model derived above, we find a formula for $m=1$.

Since $F(1, n)$ denotes the number of areas, created by either enclosed or infinite $\mathrm{n}$ cuts in one dimension, $F(1, n)$ will fulfill

$$
\left\{\begin{array}{l}
F(1,1)=2 \\
F(1, n)-\mathrm{F}(1, n-1)=1, n \geq 2, n \in N
\end{array}\right.
$$

We list out the recursive terms

$$
\begin{aligned}
& F(1,2)-F(1,1)=1 \\
& F(1,3)-F(1,2)=1 \\
& F(1, n)-F(1, n-1)=1
\end{aligned}
$$

Adding up all the terms, we get

$F(1, n)-F(1,1)=1+1+1+\cdots+1=n-1$

So $F(1, n)=n+1$.

\subsection{2. $F(2, n)$ General Form}

\section{[Investigation]}

Again, using the mathematical model derived above and similar methods as before, we find a formula for $m=2$.

Since $F(2, n)$ denotes the number of areas, which created by either enclosed or infinite $\mathrm{n}$ cuts in two dimensions, $F(2, n)$ will fulfill

$$
\left\{\begin{array}{l}
F(2,1)=2 \\
F(2, n)-\mathrm{F}(2, n-1)=F(1, n-1)=n, n \geq 2, n \in N
\end{array}\right.
$$

Listing out all the recursive terms

$$
\begin{aligned}
& F(2,2)-F(2,1)=2 \\
& F(2,3)-F(2,2)=3 \\
& F(2, n)-F(2, n-1)=n
\end{aligned}
$$

Adding all of the terms, we get

$$
F(2, n)-F(2,1)=2+3+4+\cdots+n=\frac{(n+2)(n-1)}{2}
$$

Thus, $F(2, n)=\frac{n^{2}+n-2}{2}+2=\frac{n^{2}+n+2}{2}$. 


\subsection{3 $F(3, n)$ General Form}

\section{[Investigation]}

Similarly, we find a formula for $m=3$.

Since $F(3, n)$ denotes the number of areas, in either enclosed or infinite n planar cuts and in three dimensions,

$F(3, n)$ will fulfill: $\left\{\begin{array}{l}F(3,1)=2 \\ F(3, n)-\mathrm{F}(3, n-1)=F(2, n-1)=\frac{n^{2}-n+2}{2}, n \geq 2, n \in N\end{array}\right.$

Listing out the recursive terms

$F(3,2)-F(3,1)=2$

$F(3,3)-F(3,2)=4$

$F(3, n)-F(3, n-1)=\frac{n^{2}+n+2}{2}$

Adding the terms, we get

$F(3, n)-F(3,1)=2+4+7+\cdots+\frac{n^{2}+n+2}{2}=\frac{n^{3}+5 n-6}{6}$

Thus, $F(3, n)=\frac{n^{3}+5 n-6}{6}+2=\frac{n^{3}+5 n+6}{6}$.

\subsection{4 $F(m, n)$ General Formula Prediction}

Using the previously obtained formulas for $F(1, n), F(2, n), F(3, n)$, a pattern was noticed and used to calculate $F(4, n)$ and $F(5, n)$ using the form $C_{m}^{n}$ :

$F(1, n)=n+1=C_{1}^{n+1}, n \in N$

$F(2, n)=\frac{n^{2}+n+2}{2}=C_{2}^{n+1}+C_{0}^{n+1}, n \in N$

$F(3, n)=\frac{n^{3}+5 n+6}{6}=C_{3}^{n+1}+C_{1}^{n+1}, n \in N$

$\mathrm{F}(4, n)=\frac{n^{4}-2 n^{3}-n^{2}+14 n+36}{24}=C_{4}^{n+1}+\mathrm{C}_{2}^{\mathrm{n}+1}+\mathrm{C}_{0}^{n+1}, n \in N$

$\mathrm{F}(5, n)=\frac{n^{5}-5 n^{4}+25 n^{3}+5 n^{2}+94 n+120}{120}=C_{5}^{n+1}+\mathrm{C}_{3}^{\mathrm{n}+1}+\mathrm{C}_{1}^{n+1}, n \in N$ 
There is an evident pattern for the generalized forms above. Applying that pattern, the general formula is predicted as: $F(m, n)$ general formula

(1)When $m$ is odd, $F(m, n)=C_{m}^{n+1}+C_{m-2}^{n+1}+C_{m-4}^{n+1}+\cdots+C_{1}^{n+1}$ and $m, n \in N, m \leq n$.

(2) When $m$ is even, $F(m, n)=C_{m}^{n+1}+C_{m-2}^{n+1}+C_{m-4}^{n+1}+\cdots+C_{0}^{n+1}$ and $m, n \in N, m \leq n$.

\section{[Proof]}

Proof by induction and casework:

(1) When $m=1$, the equation $F(1, n)=n+1=C_{1}^{n+1}$ holds

When $m=2$, the equation $F(2, n)=\frac{n^{2}+n+2}{2}=C_{2}^{n+1}+C_{0}^{n+1}$ holds.

(2) Assume that when $m=k$ and $k$ is an odd number, the equation $F(k, n)=C_{k}^{n+1}+C_{k-2}^{n+1}+\cdots+C_{1}^{n+1}$ holds.

Assume that when $m=k$ and $k$ is an even number, the equation $F(k, n)=C_{k}^{n+1}+C_{k-2}^{n+1}+\cdots+C_{0}^{n+1}$ holds.

Then when $m=k+1$,

(i) Case 1: $k$ is an odd number, $k+1$ is even

$$
\begin{aligned}
& F(k+1, n)=[F(k+1, n)-F(k+1, n-1)]+[F(k+1, n-1)-F(k+1, n-2)]+\cdots+ \\
& {[F(k+1,2)-F(k+1,1)]+F(k+1,1)} \\
& =F(k, n-1)+F(k, n-2)+F(k, n-3)+\cdots+F(k, 1)+F(k+1,1) \\
& =\sum_{i=1}^{n-1} F(k, i)+2 \\
& =\sum_{i=1}^{n-1}\left[C_{k}^{i+1}+C_{k-2}^{i+1}+\cdots+C_{1}^{i+1}\right]+2 \\
& =C_{k+1}^{n+1}+C_{k-1}^{n+1}+\cdots+C_{2}^{n+1}+C_{0}^{n+1}
\end{aligned}
$$

(ii) Case 2: $k$ is an even number, $k+1$ is odd

$$
F(k+1, n)=[F(k+1, n)-F(k+1, n-1)]+[F(k+1, n-1)-F(k+1, n-2)]+\cdots+
$$




$$
\begin{aligned}
& {[F(k+1,2)-F(k+1,1)]+F(k+1,1)} \\
& =F(k, n-1)+F(k, n-2)+F(k, n-3)+\cdots+F(k, 1)+F(k+1,1) \\
& =\sum_{i=1}^{n-1} F(k, i)+2 \\
& =\sum_{i=1}^{n-1}\left[C_{k}^{i+1}+C_{k-2}^{i+1}+\cdots+C_{0}^{i+1}\right]+2 \\
& =C_{k+1}^{n+1}+C_{k-1}^{n+1}+\cdots+C_{3}^{n+1}+C_{1}^{n+1}
\end{aligned}
$$

Thus, the pattern holds for $m=k+1$.

Therefore, through proof by induction, we know the general formula for $F(m, n)$ :

(1)If $m$ is odd, $F(m, n)=C_{m}^{n+1}+C_{m-2}^{n+1}+C_{m-4}^{n+1}+\cdots+C_{1}^{n+1}$, and $m, n \in N, m \leq n$

(2)If $m$ is even, $F(m, n)=C_{m}^{n+1}+C_{m-2}^{n+1}+C_{m-4}^{n+1}+\cdots+C_{0}^{n+1}$, and $m, n \in N, m \leq n$

Our predicted general formula has been proved and can be established as a theorem.

\section{[Theorem 2]}

In a $m$ dimensional $(m \in N, m \geq 2)$ space, given $n(m-1)$ dimensional cuts, there will be a maximum of $F(m, n)$ enclosed or infinite fields total, where $F(m, n)$ can be expressed as:

$$
\begin{aligned}
& \text { (1)If } m_{\text {is odd, }} F(m, n)=C_{m}^{n+1}+C_{m-2}^{n+1}+C_{m-4}^{n+1}+\cdots+C_{1}^{n+1} \text {, and } m, n \in N, m \leq n \\
& \text { (2)If } m_{\text {is even, }} F(m, n)=C_{m}^{n+1}+C_{m-2}^{n+1}+C_{m-4}^{n+1}+\cdots+C_{0}^{n+1} \text {, and } m, n \in N, m \leq n
\end{aligned}
$$

\subsection{Finding Another Mathematical Model to Prove the General Formula for $f(m, n)$ and $F(m, n)$}

Now let's find another mathematical model for $\mathrm{f}(\mathrm{m}, \mathrm{n})$. In our 1st mathematical model, we found that there was a relation between $f(m, n)$ and $n$. As $n$ increases the value of $f(m, n)$ also changes. Additionally, not only is there a change in values there is also a change in the number of intersections, lines and faces. This leads us to ponder if another mathematical model could be used to represent the number of intersections, lines and faces. This is similar to Euler's Formula (Song, 1998), which consists of vertices, edges and faces. We wish to show through Euler's Formula, a mathematical model for $\mathrm{f}(\mathrm{m}, \mathrm{n})$.

The other mathematical model--- Modified Euler's Formula---- Applied and used to obtain Theorem 3 and 4 of this project, proved below:

\section{[Theorem 3]}

In a two-dimensional connected enclosed graph, the graph will satisfy the Modified Euler's Equation: V-E+F=1, where $\mathrm{V}$ is the number of vertices, $\mathrm{E}$ is the number of enclosed edges, and $\mathrm{F}$ is the number of enclosed faces in the graph.

\section{[Proof]}

Assume that the 2-D enclosed connected graph has n regions, namely $F_{1}, \mathrm{~F} 2, \ldots . ., \mathrm{Fn}$, let $\mathrm{F} 1$ be the first enclosed region, the number of edges be E1, and the number of vertices be V1. We can immediately conclude that E1=V1. 
Let's look at the second enclosed area, F2. After subtracting the number of edges, the second enclosed area has, to E1, we get E2 and we do this similarly for V2. Because the two enclosed areas share an edge and two vertices, we get the following relationship between $\mathrm{E}$ and $\mathrm{V}: \mathrm{E} 2=\mathrm{V} 2+1$.

Let's look at the third enclosed area, F3. It has the leftover edge not used in F1or F2, called E3, and the number of vertices V3.

This should be divided into two cases:

(1) If $\mathrm{F} 3$ and $F_{1} \cup F_{2}$ have one edge in common, then $F_{3}$ and $F_{1} \cup F_{2}$ will have two vertices in common. Hence,

$E_{3}=V_{3}+1$

(2) If $\mathrm{F} 3$ and $F_{1} \cup F_{2}$ have two edges in common, then $\mathrm{F} 3$ and $F_{1} \cup F_{2}$ will have 3 vertices in common since 3

vertices makes two edges. So

$E_{3}=V_{3}+1$

We can conclude that for a third enclosed area, $E_{3}=V_{3}+1$.

Moving on to the fourth enclosed area F4, the number of edges not used would be E4 and the number of vertices would be V4.

This can then be divided into 3 cases:

Case 1: If F4 and $F_{1} \cup F_{2} \cup F_{3}$ have one common edge, then F4 and $F_{1} \cup F_{2} \cup F_{3}$ will share 2 vertices, which leads to the equation $E_{4}=V_{4}+1$.

Case 2: If F4and $F_{1} \cup F_{2} \cup F_{3}$ have two edges in common, then F4 and $F_{1} \cup F_{2} \cup F_{3}$ will have three shared vertices and have an equation of $E_{4}=V_{4}+1$.

Case 3: If F4 and $F_{1} \cup F_{2} \cup F_{3}$ have three common edges, then F4 and $F_{1} \cup F_{2} \cup F_{3}$ will have four shared vertices which results in $E_{4}=V_{4}+1$.

We conclude that $E_{4}=V_{4}+1$.

Finally, to prove the general form, we look at the nth region $F_{n}$, the extra edges would be $E_{n}$, and the total vertices are $V_{n}$.

We want to show that $E_{n}=V_{n}+1$.

As from above, when the value of $\mathrm{F}$ is $\mathrm{n}$, the value $\mathrm{V}=V_{1}+V_{2}+V_{3}+\cdots+V_{n}$, the value of $\mathrm{E}=$

$E_{1}+E_{2}+E_{3}+\cdots+E_{n}$, and the value of V-E+F is $\left(V_{1}+V_{2}+V_{3}+\cdots+V_{n}\right)-\left(E_{1}+E_{2}+E_{3}+\cdots+E_{n}\right)+n$

$=-(n-1)+n=1$.

\section{[Theorem 4]}


In a two-dimensional connected enclosed graph, the graph will satisfy the Modified Euler's Equation for Unrestricted Areas, $V-E+F=1$, where $\mathrm{V}$ is the number of vertices, $\mathrm{E}$ is the number of edges (finite or infinite), and $\mathrm{F}$ is the number of faces (enclosed or infinite).

[Proof]

The addition of linear cuts constructed to transform all infinite areas into enclosed fields will result in the addition of many lines and fields. Let us assume that after $n$ cuts, all infinite areas will become enclosed fields. In this newly formed, enclosed connected graph, let $V^{\prime}$ be the number of vertices, $E^{\prime}$ be the number of finite edges, and $F^{\prime}$ be the number of finite faces. In the original enclosed connected graph, the number of vertices $\mathrm{V}$ will equal $\mathrm{V}^{\prime}$, the number of edges $\mathrm{E}$ will equal $E^{\prime}-n$, the number of faces $\mathrm{F}$ will equal $F^{\prime}-n$. Thus,

$V-E+F=V^{\prime}-\left(E^{\prime}-n\right)+\left(F^{\prime}-n\right)=V^{\prime}-E^{\prime}+F^{\prime}=1$.

2.5.1 Applying the Second Mathematical Method, We Confirm the General Formulas for $f(2, n)$ and $F(2, n)$

\section{[Original Problem Alternate Solution]}

An infinite pizza is cut $n$ times, and every cut is straight, prove that the maximum value of pizzas slices resulting from $n$

cuts is $\frac{(n-1)(n-2)}{2}$.

\section{[Proof]}

Since $\mathrm{n}$ cuts do not have any that are parallel cuts,

It is reasonable to say that of the $\mathrm{n}$ cuts, each cut will intersect with all other cuts.

This means that each line will have $n-2$ line segments and $n-1$ vertices, because each edge is made up of 2 vertices.

We know that $V=\frac{n(n-1)}{2}$, and $E=n^{2}-2 n$. By Theorem Two, $F=E+1-V=\left(n^{2}-2 n\right)+1-\frac{n(n-1)}{2}$

[Rederiving $F(2, n)$ ]

On a two dimensional plane, given $n$ non-parallel cuts, the maximum number of areas, enclosed and infinite, is

$$
\frac{n^{2}+n+2}{2}
$$

\section{[Prove]}

Since each of the $n$ cuts intersects with all other cuts, each cut will consist of $n-1$ point of intersections, with two

cuts sharing each intersection. Thus, $V=\frac{n(n-1)}{2}, E=n^{2}$. By Theorem 4,

$$
F=E-1+V=n^{2}+1-\frac{n(n-1)}{2}=\frac{n^{2}+n+2}{2} .
$$

\subsubsection{Extension 1 of Original Problem}

An infinite pizza is cut $\mathrm{n}$ times. Each cut is curved. Each curved line could intersect at 2 points. Prove that the

maximum number of pizzas one can get using $n$ cuts is $\frac{2 n^{2}-3 n+2}{2}$ small pizzas.

[Proof] 
In topology, straight line is the same as a curved line because they both represent a pathway between two points so let us make the parabolic lines straight and $n$ cuts such that no cut is parallel to another. Then we could say that for any cut, it intersects with n-1 lines in the circle and every line has $2 n-2$ lines and $2 n-1$ vertices.

We get that $V=\frac{n(2 n-1)}{2}, E=n(2 n-2)$. By Theorem 2,

$F=E+1-V=\left(2 n^{2}-n\right)+1-\frac{n(2 n-1)}{2}=\frac{2 n^{2}-3 n+2}{2}$.

\subsubsection{Extension 2 of Original Problem}

An infinite pizza is cut $n$ times by straight lines. (1) When $\mathrm{n}$ cuts have $p, p \geq 2$ lines which are parallel and there does not exist three cuts that are concurrent, the maximum number of pieces of pizza that result from these $\mathrm{n}$ cuts is $\frac{(n+p)(n-p+1)+2}{2}$. (2) If of the $\mathrm{n}$ cuts, there are q cuts such that these q cuts all intersect at the same points, all other intersections are intersections of exactly two lines, no two lines are parallel, the maximum number of areas resulting from $n$ cuts is $\frac{(n+1)(n-1+1)+2 k}{2}$.

\section{[Proof]}

(1) Of the $p$ lines that are parallel, each line will have $n-p$ intersections. In addition, the unparalleled lines all have n-1 intersections.

This means that the total number of edges will be: $E=p(n-p+1)+(n-p) n=(n-p)(n+p)+p$ and since there are no three lines that are concurrent, the total number of vertices is

$V=\frac{1}{2}[p(n-p)+(n-p)(n-1)]=\frac{(n-p)(n+p-1)}{2}$.

According to Theorem 2, we can determine that:

$$
\begin{aligned}
& F=E+1-V=(n-p)(n+p)+p+1-\frac{(n-p)(n+p-1)}{2} \\
& =\frac{(n+p)(n-p+1)+2}{2}
\end{aligned}
$$

(2) Excluding the shared point shared by $q$ cuts, each line of the q lines has $n-1$ vertices. In addition, of the $n-q$ cuts, every cut has n-1 vertices, which means the total number of vertices is

$$
V=\frac{1}{2}[q(n-q)+(n-q)(n-1)]+1=\frac{(n-q)(n+q-1)+2}{2}
$$

and the total number of line segment/edges is $E=q(n-q+2)+(n-q) n=(n-q)(n+q)+2 q$. 
Using Theorem 1, we can determine that $F=E+1-V=(n-q)(n+q)+2 q+1-\frac{(n-q)(n+q-1)+2}{2}$

$=\frac{(n+q)(n-q+1)+2 q}{2}$.

2.5.4 Use the Second Mathematical Model to Find the General Form of $f(3, n)$ and $F(3, n)$.

Using the formula from Mathematical Model 2---[Euler's Formula]we confirm the formulas of $f(3, n)$ and $F(3, n)$.

\section{[Theorem 5]}

If in space there is a 3-D connected graph which contains the number of vertices $\mathrm{V}$, the number of finite edges $\mathrm{E}$, the number of finite faces $\mathrm{F}$, and the number of finite solids $\mathrm{S}$, then $V, E, F, S$ satisfy the equation $V-E+F-S=1$.

\section{[Proof]}

We split the problem into two cases:

(1)When $S=0$ all the planar cuts are either parallel or have the same intersection (which is a line). E, V, and F are very hard to count at this point. Therefore, we first construct a plane that is perpendicular to all shared intersections. On this plane, all preexisting planar cuts will show up as lines, all intersections of preexisting planar cuts will show up as points, and all enclosed solids will show up as enclosed areas. In order to determine the number of edges, faces, and solids of the original planar cuts, we need only to count the edges, vertices, and faces of the additional plane.

From Theorem 3, we know that $V-E+F=1$ in this additional plane. Therefore, it can determine that the Modified Euler's Formula for three dimensions is $V-E+F-S=(V-E-F)+S=1-0=1$.

(2) When $S \neq 0$, a sphere that encompasses all vertices can be constructed, creating an enclosed connected graph on the surface of the sphere. Through the use of topological techniques, the surface of the sphere can be transformed into a polyhedron solid.

Notice that the edges, faces and solids in the original three-dimensional enclosed connected graph projected onto the surface of the sphere will become points, edges, and faces respectively. We denote these as $V^{\prime}, E^{\prime}$, and $F^{\prime}$ respectively. Once the sphere has been topologically transformed into a polyhedron solid, we can apply the original Euler's Formula to get $V^{\prime}-E^{\prime}+F^{\prime}=2$. We want to prove the Modified Euler's formula in three dimensions from the above equation.

Since our Modified Euler's formula only includes enclosed edges, faces and solids, all infinite edges need to be removed. If we let V, E, F, and S represent the total amount of vertices, edges, faces, and solids respectively from the original enclosed connected graph (including all infinite fields), the number of enclosed vertices, edges, faces, and solids can be expressed as: $V-\mathrm{V}^{\prime}, E-E^{\prime}, F-F^{\prime}+1, S$

By the Poincare characteristic of polyhedrons, we get $\left(V-V^{\prime}\right)-\left(E-E^{\prime}\right)+\left(F-F^{\prime}+1\right)-S=0$

and $(V-E+F-S)-\left(V^{\prime}-E^{\prime}+S^{\prime}\right)+1=0$

Therefore, $V-E+F-S=1$.

\section{[Theorem 6]}

In a three-dimensional connected graph which contains $\mathrm{V}$ number of vertices, $\mathrm{E}$ infinite and enclosed edges, $\mathrm{F}$ infinite and enclosed faces, and $\mathrm{S}$ infinite and enclosed solids, the graph will satisfy the equation:

$V-E+F-S=-1$.

\section{[Proof]}

We split the problem into two cases:

(1) When $V=0$, all planar cuts are either parallel or have the same intersection (which is a line). E, V, and F are hard to count at this point. Therefore, we construct a plane that is perpendicular to all shared intersections. On this plane, all preexisting planar cuts will show up as lines, all intersections of preexisting planar cuts will show up as points, and all enclosed solids will show up as enclosed areas. In order to determine the number of edges, faces, and solids of the original planar cuts, we need only to count the edges, vertices, and faces of the additional plane, denoted as $\mathrm{E}, \mathrm{V}$, and $\mathrm{S}$ respectively. 
By Theorem 4, $E-F+S=1$.

Thus, $V-E+F-S=V-(E-F+S)=0-1=-1$.

(2) When $V \neq 0$, a sphere that encompasses all vertices can be constructed, creating an enclosed connected graph on the surface of the sphere. Through the use of topological techniques, the surface of the sphere can be transformed into a polyhedron solid.

Notice that the edges, faces, and solids in the original three dimensional connected graphs once projected onto the surface of the sphere will become points, edges, and faces, respectively. We denote theses as $E^{\prime}, F^{\prime}$, and $S^{\prime}$, respectively. Once the sphere has been topologically transformed into a polyhedron solid, we can apply the original Euler's Formula to get $E^{\prime}-F^{\prime}+S^{\prime}=2$. Now, we want to prove the Modified Euler's Formula for Unrestricted Area in three dimensions from the above equation.

Since our Modified Euler's Formula for Unrestricted Area only includes enclosed edges, faces, and solids, all infinite edges need to be removed. If We let V, E, F, and S represent the total amount of vertices, edges, faces, and solids respectively from the original connected graph, the number of enclosed vertices, edges, faces, and solids can be expressed as: V,E- E', F- F', S-S'+1.

By the Poincare characteristic of polyhedrons, we get

$V-\left(E-E^{\prime}\right)+\left(F-F^{\prime}\right)-\left(S-S^{\prime}+1\right)=0$ and $(V-E+F-S)-\left(E^{\prime}-F^{\prime}+S^{\prime}\right)-1=0$.

Therefore, $V-E+F-S=-1$.

2.5.5 Rederiving $f(3, n)$

There exists an infinite cake solid divided by $n$ planar cuts. Prove that the maximum number of enclosed regions

created by $n$ cuts is $\frac{(n-1)(n-2)(n-3)}{6}$.

\section{[Proof]}

Since on each planar cut, there will be $n-1$ lines (caused by intersections with $n-1$ other planar cuts), such that no two lines are parallel, and no three lines are concurrent, these lines will create a two-dimensional enclosed connected graph. Thus, the number of vertices, finite edges, and enclosed faces on each planar cut denoted as $V_{0}, E_{0}, F_{0}$ respectively on this two-dimensional enclosed connected graph will satisfy the following:

$$
V_{0}=\frac{(n-1)(n-2)}{2}, E_{0}=(n-1)^{2}-2(n-1), F_{0}=\frac{(n-2)(n-3)}{2}
$$

The total number of vertices, finite edges, and enclosed faces will be

$$
V=\frac{1}{3} n V_{0}=\frac{n(n-1)(n-2)}{6}, E=\frac{n}{2} E_{0}=\frac{n\left(n^{2}-4 n+3\right)}{2}, F=n F_{0}=\frac{n(n-2)(n-3)}{2}
$$

By Theorem 6, we can determine that

$$
S=V-E+F+1=\frac{n(n-1)(n-2)}{6}-\frac{n(n-1)^{2}}{2}+\frac{n\left(n^{2}-n+2\right)}{2}+1=\frac{n^{3}+5 n+6}{6}
$$

\subsubsection{Rederiving $F(3, n)$}

There exists an infinite cake solid divided by $n$ planar cuts. Prove that the maximum regions created by $\mathrm{n}$ cuts is 
$\frac{\left(n^{3}+5 n+6\right)}{6}$ pieces of cake

\section{[Proof]}

Since on each planar cut, there will be n-1 lines (caused by intersections with n-1 other planar cuts), such that no two lines are parallel, and no three lines are concurrent, these lines will create a two-dimensional enclosed connected graph.

Thus, the total number of vertices, edges, and faces denoted as $V_{0}, E_{0}, F_{0}$ respectively on this two-dimensional enclosed connected graph will satisfy the following: $V_{0}=\frac{(n-1)(n-2)}{2}, E_{0}=(n-1)^{2}$,

$$
F_{0}=\frac{1}{2}\left[(n-1)^{2}+(n-1)+2\right]=\frac{n^{2}-n+2}{2}
$$

The total number of vertices, edges, and faces will be

$$
\begin{aligned}
& V=\frac{1}{3} n V_{0}=\frac{n(n-1)(n-2)}{6}, E=\frac{n}{2} E_{0}=\frac{n(n-1)^{2}}{2}, \\
& F=n F_{0}=\frac{n\left(n^{2}-n+2\right)}{2}
\end{aligned}
$$

By Theorem 3, we can determine that

$$
\begin{aligned}
& S=V-E+F+1 \\
& =\frac{n(n-1)(n-2)}{6}-\frac{n(n-1)^{2}}{2}+\frac{n\left(n^{2}-n+2\right)}{2}+1 \\
& =\frac{n^{3}+5 n+6}{6} .
\end{aligned}
$$

\section{Research Consequence and Discussion}

[Mathematical Model 1]---Generalized Recursive Rule

1. In $\mathrm{m}$ dimensions, the expression $f(\mathrm{~m}, n)$ represents the maximum number of enclosed fields in $\mathrm{m}$ dimensions given

n cuts. $f(\mathrm{~m}, n)$ will fulfill the following: $\left\{\begin{array}{l}f(m, 1)=0 \\ f(m, n)-f(m, n-1)=f(m-1, n-1), n \geq 2, n \in N\end{array}\right.$

2. In $\mathrm{m}$ dimensions, the expression $F(\mathrm{~m}, n)$ where $m \in N, m \geq 2$, represents the maximum number of enclosed fields in $\mathrm{m}$ dimensions given $\mathrm{n}$ cuts. $F(\mathrm{~m}, n)$ will fulfill the following:

$$
\left\{\begin{array}{l}
F(m, 1)=2 \\
F(m, n)-\mathrm{F}(m, n-1)=F(m-1, n-1), n \geq 2, n \in N
\end{array}\right.
$$


[Mathematical Model 2]----Modified Euler's Formula

1. In a two dimensional connected enclosed graph, the graph will satisfy the Modified Euler's Equation: $\mathrm{V}-\mathrm{E}+\mathrm{F}=1$, where $\mathrm{V}$ is the number of vertices, $\mathrm{E}$ is the number of enclosed edges, and $\mathrm{F}$ is the number of enclosed faces in the graph.

2. If in space there is a 3-D connected graph which contains the number of vertices $\mathrm{V}$, the number of finite edges $\mathrm{E}$, number of finite faces $\mathrm{F}$, and number of finite solids $\mathrm{S}$, then $V, E, F, S$ satisfy the equation $V-E+F-S=1$.

\section{[Theorem 1]}

In an infinite $m$ dimensional space $(m \in N, m \geq 2)$, given $n(m-1)$-dimensional cuts (all of which are infinite

fields), the maximum number of enclosed fields $f(m, n)$ is $f(m, n)=C_{m}^{n-1}$ where $m, n \in N$ and $m \leq n$.

\section{[Theorem 2]}

In a $m$ dimensional ( $m \in N, m \geq 2)$ space, given $n(m-1)$-dimensional cuts, there will be a maximum of

$F(m, n)$ enclosed or infinite fields total, where $F(m, n)$ can be expressed as:

(1)If $m$ is odd, $F(m, n)=C_{m}^{n+1}+C_{m-2}^{n+1}+C_{m-4}^{n+1}+\cdots+C_{1}^{n+1}$, and $m, n \in N, m \leq n$

(2)If $m$ is even,

$$
F(m, n)=C_{m}^{n+1}+C_{m-2}^{n+1}+C_{m-4}^{n+1}+\cdots+C_{0}^{n+1}, \text { and } m, n \in N, m \leq n
$$

\section{[Theorem 3]}

In a two-dimensional connected enclosed graph, the graph will satisfy the Modified Euler's Equation, V-E+F=1, where $\mathrm{V}$ is the number of vertices, $\mathrm{E}$ is the number of enclosed edges, and $\mathrm{F}$ is the number of enclosed faces in the graph.

\section{[Theorem 4]}

In a two-dimensional connected enclosed graph, the graph will satisfy the Modified Euler's Equation for Unrestricted Areas, $V-E+F=1$, where $\mathrm{V}$ is the number of vertices, $\mathrm{E}$ is the number edges (finite or infinite), and $\mathrm{F}$ is the number of faces (enclosed or infinite).

\section{[Theorem 5]}

If in space there is a 3-D connected graph which contains the number of vertices $\mathrm{V}$, the number of finite edges $\mathrm{E}$, number of finite faces $\mathrm{F}$, and number of finite solids $\mathrm{S}$, then $V, E, F, S$ satisfy the equation $V-E+F-S=1$.

\section{[Theorem 6]}

In a three dimension connected graph which contains $\mathrm{V}$ number of vertices, $\mathrm{E}$ infinite and enclosed edges, $\mathrm{F}$ infinite and enclosed faces, and $\mathrm{S}$ infinite and enclosed solids, the graph will satisfy the equation, $V-E+F-S=-1$.

\section{Conclusions and Applications}

\subsection{Conclusions}

1. This study uses the recursive relationship to prove the general formula for the maximum number of enclosed fields in two, three, and four dimensions. From the pattern of the general formulas, the general formula for $m_{\text {dimensions was }}$ predicted and proven by induction. Similarly, the general formula for $F(m, n)$ was proven.

2. A second mathematical model, the Modified Euler's Formula, was found through experimenting with vertices, faces, and edges created by $f(2, n)$ and $F(2, n): V-E+F=1$. Using the same method, the relationship between the number of vertices, edges, faces and solids created by $f(3, n)$ and $F(3, n)$ in three dimensions was found.

3. The use of the two mathematical models enabled the derivation of the general form of $f(m, n)$ and $F(m, n)$. These forms were eventually proven through induction. After finding the Euler relationship in two and three dimensions, for future work, we would like to define the Modified Euler's Formula for higher dimensions. 


\subsection{Applications}

The verification of the general formulas using the Modified Euler's formula for two dimensions and three dimensions involves tedious, and intricate calculations that can be prone with mistakes. However, accuracy in calculations verifying formulas can be improved if the steps for verification and the recursive rule were programmed into a computer, letting the computer run the calculations or catch mistakes.

Using the general formulas for $f(m, n)$ and $F(m, n)$, extend the Modified Euler's formula to $m$ dimensions by exploring the relationship between $u_{0}, u_{1}, u_{2} \ldots u_{n}$ where $u_{i}$ is the number of I-dimensional objects created in the connected graphs of $f(m, n)$ and $F(m, n)$ to see if they will satisfy the condition $u_{0}-u_{1}+u_{2} \ldots(-1)^{n} u_{n}=1$.

\subsection{Discussions}

After looking at the PUMaC 2014 \#2 pizza problem, we immediately thought about changing the dimensions to see if there was any relation. We started working with the 1st mathematical model of recursive cuts and then looked at what happens when we cut in a 1-D plane with points. The relation was that when each time a cut was made, the number of lines increased by 1 . Noting that relation, we moved on to cutting in the 2 -dimensional plane. The relation in the 2-dimensional plane was actually a recursive rule. This makes sense because in the 2-dimensional plane, two lines intersect to form a point, which in turn is the cut we did in the 1-dimensional plane. We inferred that the same recursion applied when we cut in the 3-dimensional plane. Because when planes (each cut in the 3rd dimension is a plane) intersect, they become lines (each cut in the 2-dimensional plane). Noting this relation, we then tackled the general form of $f(m, n)$ and wrote $f(m, n)$ as the sum of several combinatorial terms. We noticed that there could possibly be another mathematical model that solidifies $\mathrm{f}(\mathrm{m}, \mathrm{n})$ 's general form, and that was using a modified Euler's formula. This was because when we were cutting, we were paying attention to the cuts, which could be looked at as edges of the regions cut out. First we proved that our modified Euler's formula worked in a 2-dimensional plane, then applied it to find the general form of $\mathrm{f}(2, \mathrm{n})$. After several extensions, we proved that our modified Euler's formula worked in a 3 -dimensional plane and applied it to derive the general form of $\mathrm{f}(3, \mathrm{n})$. After deriving the general form, our results in the mathematical model of Euler's formula agreed with the results of our first mathematical model, which was recursive cutting. Finally, we summarized the whole project by stating the general forms of $\mathrm{f}(\mathrm{m}, \mathrm{n})$ where the general form was dependent of the parity of $\mathrm{m}$.

The major mathematical subjects used in this project include Euler's Formula, recursive rule, and finite field. They were used to find if there was a relation between the increasing dimensions, the number of regions formed by the cuts and the number of cuts. They were also used to find a general formula for $\mathrm{f}(\mathrm{m}, \mathrm{n})$. The findings of this project could be applied to several scenarios as listed below. If there was a meteor that was going to hit Earth, what is the number of cuts needed to decimate the meteor and save Earth? If all the food on Earth turned into a giant object floating in the sky, what is the least number of cuts needed to satisfy the hungry population of Earth? The results of this project could blossom into many more miraculous relations and thereby showcasing the beauty, the endless surprises and possibilities in the world of math.

\section{References}

Lazy caterer's sequence (2017). In Wikipedia, the free encyclopedia. Retrieved from https://en.wikipedia.org/wiki/Lazy_caterer's_sequence

PUMaC (2014). PUMaC 2014 Combinatorics $A$. retrieved from https://pumac.princeton.edu/wp-content/uploads/2014/11/2014-Combinatorics-A-Problems.pdf

Puzzling Stack Exchange (2014). Maximum number of pieces of equal area you can obtain by cutting a certain number of times. Retrieved from https://puzzling.stackexchange.com/questions/3680

Simone, C. D., \& Rinaldi, G. (2008). A cutting plane algorithm for the max-cut problem. Optimization Methods and Software, 3, 195-214. http://dx.doi.org/10.1080/10556789408805564

Song, B. S. (1998). From Euler's equation to dimensional planes cutting, Mathematics promotion, 2(3), 54-60.

\section{Copyrights}

Copyright for this article is retained by the author(s), with first publication rights granted to the journal.

This is an open-access article distributed under the terms and conditions of the Creative Commons Attribution license (http://creativecommons.org/licenses/by/4.0/). 